\title{
Influence of cryopreservation on perinatal outcome after blastocyst- $v$ s cleavage-stage embryo transfer: systematic review and meta-analysis
}

\author{
C. ALVIGGI ${ }^{1}$, A. CONFORTI ${ }^{1}$,, I. F. CARBONE${ }^{2}$, R. BORRELLI $^{1}$, G. DE PLACIDO $^{1}$ \\ and S. GUERRIERO ${ }^{3}$ (i) \\ ${ }^{1}$ Department of Neuroscience, Reproductive Medicine, Odontostomatology, University of Naples Federico II, Naples, Italy; ${ }^{2}$ Department of \\ Obstetrics and Gynecology, Mangiagalli, Fondazione IRCCS Ca' Granda, Ospedale Maggiore Policlinico, Milan, Italy; ${ }^{3}$ Department of \\ Obstetrics and Gynecology, University of Cagliari, Policlinico Universitario Duilio Casula, Monserrato, Cagliari, Italy
}

KEYWORDS: ART; blastocyst; cleavage-stage embryos; fresh cycle; frozen cycle; IVF; perinatal outcome

\begin{abstract}
Objective To compare the perinatal outcomes of singleton pregnancies resulting from blastocyst- vs cleavage-stage embryo transfer and to assess whether they differ between fresh and frozen embryo transfer cycles.
\end{abstract}

Methods A systematic review of the literature was carried out using the Scopus, MEDLINE and ISI Web of Science databases with no time restriction. We included only peer-reviewed articles involving bumans, in which perinatal outcomes of singleton pregnancies after blastocyst-stage embryo transfer were compared with those after cleavage-stage embryo transfer. Primary outcomes were preterm birth before 37 weeks and low birth weight $(<2500 \mathrm{~g})$. Secondary outcomes were very preterm birth before 32 weeks, very low birth weight $(<1500 \mathrm{~g})$, small-for-gestational-age (SGA), large-for-gestational-age (LGA), perinatal mortality and congenital anomaly. A meta-analysis was performed using a random-effects model. Three subgroups were evaluated: fresh only, frozen only and fresh plus frozen embryo transfer cycles.

Results From a total of 3928 articles identified, 14 were selected for qualitativelquantitative analysis. Significantly higher incidences of preterm birth < 37weeks (11 studies, $\mathrm{n}=106629$ participants; risk ratio $(R R), 1.15$ (95\% CI, 1.05-1.25); $\mathrm{P}=0.002)$ and very preterm birth $<32$ weeks (seven studies, $\mathrm{n}=103742 ; R R, 1.16$ (95\% CI, 1.02-1.31); $\mathrm{P}=0.03$ ) were observed after blastocyst- than after cleavage-stage embryo transfer in fresh cycles. However, the risk of preterm and very preterm birth was similar after blastocyst- and cleavage-stage transfers in frozen and fresh plus frozen cycles. Overall effect size analysis revealed fewer SGA deliveries after blastocyst-compared with cleavage-stage transfer in fresh cycles but a similar number in frozen cycles. Conversely, more LGA deliveries were observed after blastocyst- compared with cleavage-stage transfer in frozen cycles (two studies, $\mathrm{n}=39044 ; \mathrm{R}, 1.18$ (95\% CI, 1.09-1.27); $\mathrm{P}<0.0001)$ and no differences between the two groups in fresh cycles (four studies, $\mathrm{n}=42$ 982; RR, 1.14 (95\% CI, 0.97-1.35); $\mathrm{P}=0.11$ ). There were no differences with respect to low birth weight, very low birth weight or congenital anomalies between blastocyst- and cleavage-stage transfers irrespective of the cryopreservation method employed. Only one study reported a higher incidence of perinatal mortality after blastocyst- vs cleavage-stage embryo transfer in frozen cycles, while no differences were found in fresh cycles.

Conclusions Our results suggest that cryopreservation of embryos can influence outcome of pregnancy conceived following blastocyst- vs cleavage-stage embryo transfer in terms of preterm birth, very preterm birth, LGA, SGA and perinatal mortality. Caution should be exercised in interpreting these findings given the low level of evidence and wide heterogeneity of the studies. Copyright (C) 2017 ISUOG. Published by John Wiley \& Sons Ltd.

\section{INTRODUCTION}

Since the first reports on the technique were published ${ }^{1,2}$, extended embryo culture has become widespread in assisted reproductive technology $y^{3,4}$. Compared with traditional cleavage-stage embryo transfer, this procedure results in a sort of 'natural selection' of the most viable embryos, analagous to the usual process during spontaneous conception ${ }^{5}$.

Correspondence to: Dr A. Conforti, Department of Neuroscience, Reproductive Medicine, Odontostomatology, University of Naples Federico II, Naples, Italy (e-mail: confale@hotmail.it)

Accepted: 25 October 2017 
Despite theoretical advantages, the clinical efficacy of blastocyst-stage $v$ s cleavage-stage transfer is debatable. In fact, while in 2016 a Cochrane meta-analysis reported increased rates of clinical pregnancy and live birth after blastocyst transfer ${ }^{6}$, a more recent meta-analysis did not find any statistically significant differences in these outcomes between blastocyst- and cleavage-stage transfer ${ }^{7}$.

Data on the perinatal outcome of babies born after blastocyst-stage embryo transfer are even more controversial $^{8-11}$. Earlier meta-analyses claimed that blastocyst transfer is associated with an increased risk of preterm birth, very preterm birth and congenital malformations ${ }^{8,11}$; however, subsequent studies have not confirmed these observations $9,10,12,13$. In fact, an analysis of 43952 singleton deliveries after transfer of blastocyst- or cleavage-stage embryos did not show an increased risk of preterm birth in pregnancies resulting from blastocyst transfer ${ }^{9}$. Similarly, a recent study of 277042 embryo-transfer cycles in Japan did not find any statistically significant increase in the risk of very preterm birth and preterm birth after blastocyst transfer ${ }^{12}$. Finally, a study evaluating the neonatal and perinatal outcomes of 30566 singleton pregnancies did not identify any statistically significant differences in congenital anomalies or preterm and very preterm birth between in-vitro fertilization (IVF) carried out with cleavage-stage embryos and IVF carried out with blastocysts ${ }^{10}$.

A limitation of previous meta-analyses on this topic is that they merged data from fresh and frozen embryo transfers and did not consider the effect of cryopreservation on the results $8,11,14$. The aim of this systematic review and meta-analysis was to compare the perinatal outcomes of singleton pregnancies resulting from blastocyst-stage embryo transfer with those resulting from cleavage-stage embryo transfer, and to assess whether they differ between fresh and frozen embryo-transfer cycles.

\section{METHODS}

Protocol, eligibility criteria, information sources and search

This systematic review was conducted according to PRISMA guidelines. An electronic search of MEDLINE (PubMed), ISI Web of Science and Scopus databases was performed, up to August 2017. The reference lists of relevant articles and reviews were also searched manually. Combinations of the following keywords and search terms were used: ('blastocyst' OR 'cleavage stage embryo') AND ('congenital abnormalities' OR 'congenital defect' OR 'deformity' OR 'birth defect' OR 'perinatal outcome' OR 'perinatal mortality' OR 'preterm birth' OR 'premature birth' OR 'birth weight'). No time or language restrictions were adopted, but the search was limited to human studies.

\section{Study selection, data collection and data items}

Three reviewers (A.C., R.B., I.F.C.) evaluated the titles and abstracts of the studies identified. Duplicate records were removed using Endnote online software (https://access.clarivate.com/\#/login?app=endnote). Disagreements were resolved by discussion between the reviewers, and consultation with the more experienced authors (C.A., S.G., G.D.). We included studies in which the perinatal outcomes of singletons born after blastocyst-stage transfer were compared with those of singletons born after cleavage-stage transfer in infertile women. Case series, case reports, books, congress abstracts and gray literature were not included in the analysis. In cases of papers with replication of data, the studies with the largest number of observations were chosen.

\section{Risk of bias, summary measures and synthesis of results}

Risk of bias and quality assessment of the included studies was performed using the Newcastle-Ottawa Scale $(\mathrm{NOS})^{15}$. Three authors (A.C., R.B., I.F.C.) independently assessed the risk of bias for each included study and conflicts were resolved by discussion with the more experienced authors (C.A, S.G., G.D.). The NOS score was used to evaluate the included studies, and each study was judged on three issues: selection of the study group, comparability between groups and ascertainment of exposed and non-exposed cohorts ${ }^{15}$. Primary outcomes were preterm birth, defined as live birth before 37 weeks' gestation, and low birth weight, defined as birth weight $<2500 \mathrm{~g}$. Secondary outcomes were: very preterm birth (live birth before 32 weeks), very low birth weight $(<1500 \mathrm{~g})$, small-for-gestational-age (SGA) neonate, large-for-gestational-age (LGA) neonate, perinatal mortality and congenital anomaly. Data were extracted independently by three reviewers (A.C., R.B., I.F.C.) and discrepancies were resolved by discussion with the more experienced authors (C.A., S.G., G.D.).

To minimize the risk of bias across studies, a comprehensive selection of the studies was conducted in order to prevent data duplication. Given the difficulty of detecting and correcting for publication bias, it was assessed by multiple analyses. Funnel plots of primary outcomes were evaluated both visually and formally with the 'trim and fill' method and the Egger test ${ }^{16,17}$.

\section{Quality of evidence}

The quality of evidence for the assessed outcomes was evaluated using the Grading of Recommendations Assessment, Development and Evaluation (GRADE) system ${ }^{18}$. Quality of evidence was assessed by evaluating the following items: limitations, inconsistency, indirectness, imprecision and risk of publication bias.

\section{Sensitivity analysis}

Sensitivity analysis was carried out by measuring the overall effect size for all groups. Studies judged to be at the highest risk of bias, namely those with the lowest NOS score, were excluded from the analysis. 


\section{Statistical analysis}

Statistical analysis was carried out using RevMan software (Review Manager version 5.3; The Nordic Cochrane Centre, The Cochrane Collaboration). Data from fresh cycles, frozen cycles and fresh plus frozen cycles were combined to obtain a pooled risk ratio (RR) using the Mantel-Haenszel method. Meta-analysis was conducted using a random-effects model. Between-study heterogeneity was addressed using $I^{2}$, which represents the percentage of total variation in the estimated effect across studies, with $I^{2}>50 \%$ indicating substantial heterogeneity; $P<0.05$ was considered to indicate statistical significance.

\section{RESULTS}

A total of 3928 articles were identified through the search (MEDLINE, 1052; ISI Web of Science, 1085; Scopus, 1791) and 420 duplications were removed using EndNote online library. The titles and abstracts of 3508 papers were scrutinized and 39 full papers were assessed for eligibility. Twenty-five papers were excluded, including two papers for which data could not be extracted ${ }^{19,20}, 16$ papers that did not fulfill our inclusion criteria and four papers in which data replication was detected ${ }^{13,21-23}$. In detail, Nakashima et al. ${ }^{22}$ Kallen et al. ${ }^{21}$ and Sazonova et $a .^{23}$ extracted newborn data from the same registry used more recently by Ginström Ernstad et al. ${ }^{10}$ and Ishihara et al. ${ }^{12}$, while Oron et al. reported similar data in two papers ${ }^{13,24}$. The other three studies ${ }^{25-27}$ were not considered because they analyzed the same population as other included studies ${ }^{10,12,24}$. Thus, 14 articles were included in the qualitative/quantitative analysis (Figure 1). Characteristics of the included studies are reported in Table 1 and the risk of bias within each study in Table 2 .

\section{Primary outcomes}

The incidence of preterm birth before 37 weeks was investigated in 13 studies $2,9,10,12,24,28-35$ (blastocyst stage, $n=90150$; cleavage stage, $n=103677$ ). A significantly higher risk of preterm birth in singletons born after blastocyst- than after cleavage-stage embryo transfer was revealed by the overall effect size (RR, 1.10 (95\% CI, $1.01-1.20) ; P=0.04, I^{2}=81 \%$ ) and subgroup analysis of fresh embryo transfer cycles (RR, 1.15 (95\% CI, $1.05-1.25) ; P=0.002, I^{2}=58 \%$ ) (Figure 2). Subgroup analysis of frozen cycles alone and fresh plus frozen cycles showed no significant difference between blastocystand cleavage-stage transfer with respect to preterm birth.

Low birth weight $(<2500 \mathrm{~g})$ was investigated in 11 studies $9,10,12,24,28-32,34,35$ (blastocyst stage, $n=87353$; cleavage stage, $n=101613$ ). The rate of low-birth-weight neonates was similar in singletons born after blastocystand cleavage-stage embryo transfer irrespective of the cryopreservation procedure used (Figure S1).

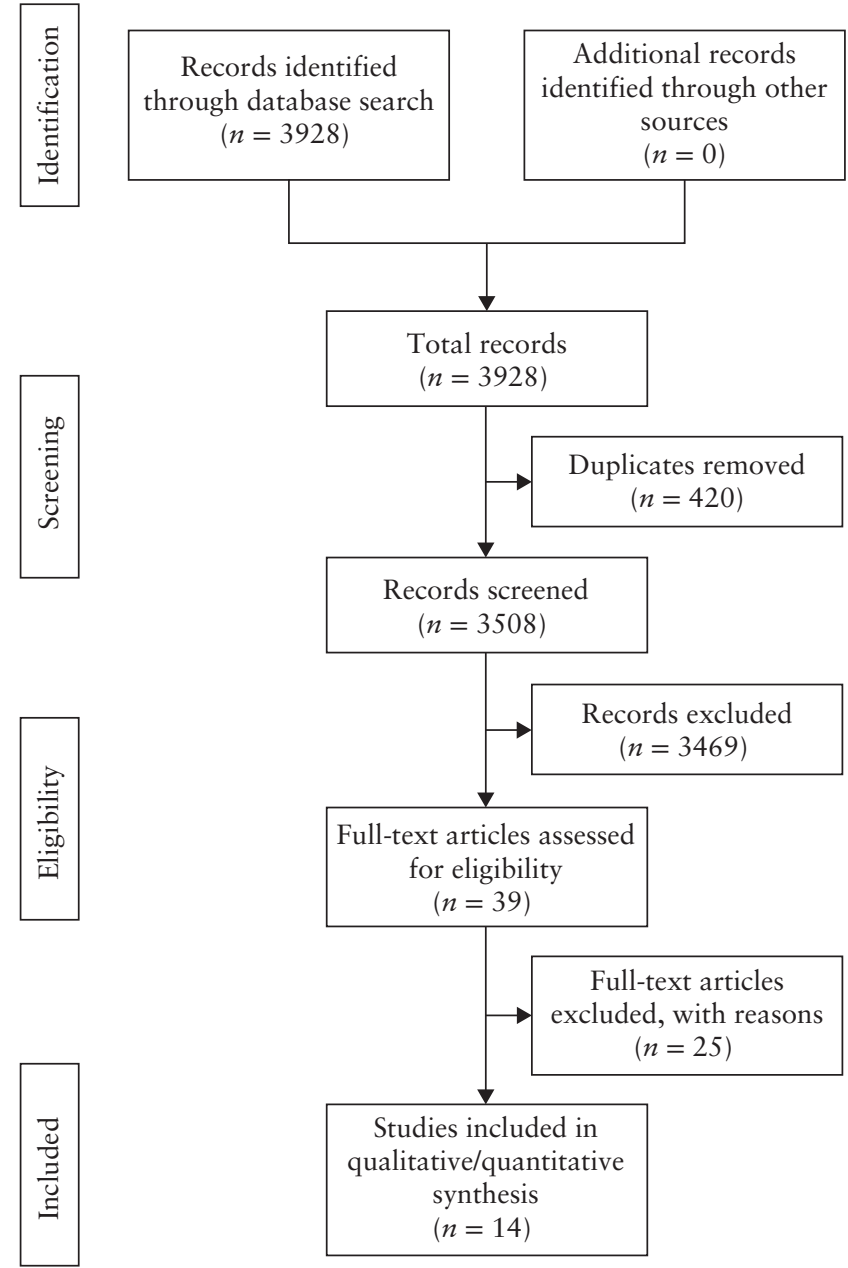

Figure 1 Flowchart summarizing identification and selection of studies included in systematic review and meta-analysis.

\section{Secondary outcomes}

The incidence of very preterm birth before 32 weeks was assessed in eight studies $2,10,12,24,28-31$ (blastocyst stage, $n=60488$; cleavage stage, $n=86500)$. The overall effect size and subgroup analysis of frozen only and fresh plus frozen cycles did not reveal a significant difference between blastocyst- and cleavage-stage embryo transfer with respect to this outcome (Figure 3). Subgroup analysis of fresh cycles showed a higher risk of very preterm birth in pregnancies resulting from blastocyst-stage compared with cleavage-stage embryo transfer (RR, 1.16 (95\% CI, $\left.1.02-1.31) ; P=0.03, I^{2}=16 \%\right)$.

SGA as a perinatal outcome was analyzed in seven studies ${ }^{9,10,12,24,30,31,36}$ (blastocyst stage, $n=83123$; cleavage stage, $n=93369$ ). The overall effect size revealed a lower risk of SGA birth in blastocyst-stage than in cleavage-stage transfers (RR, 0.84 (95\% CI, 0.76-0.92); $P=0.0002, I^{2}=64 \%$ ) (Figure 4). Similarly, a lower risk of SGA after blastocyst- $v$ s cleavage-stage embryo transfer was shown in subgroup analysis of fresh only cycles, fresh plus frozen cycles and frozen only cycles, though the difference did not reach statistical significance in the latter.

The incidence of LGA neonates was addressed in five studies ${ }^{10,12,24,30,36}$ (blastocyst stage, $n=40299$; 
Table 1 Characteristics of studies included in meta-analysis, comparing perinatal outcomes of singleton pregnancies resulting from blastocyst-stage $v$ s cleavage-stage embryo transfer

\begin{tabular}{|c|c|c|c|c|c|c|c|c|}
\hline \multirow[b]{2}{*}{ Study } & \multirow[b]{2}{*}{ Years of study } & \multirow[b]{2}{*}{ Country } & \multicolumn{2}{|c|}{ Singleton delivery (n) } & \multirow[b]{2}{*}{ Design } & \multirow[b]{2}{*}{$\begin{array}{l}\text { Type of } \\
\text { cycle }\end{array}$} & \multirow[b]{2}{*}{$\begin{array}{l}\text { Method of data } \\
\text { collection }\end{array}$} & \multirow[b]{2}{*}{$\begin{array}{l}\text { Outcome } \\
\text { analyzed }\end{array}$} \\
\hline & & & $\begin{array}{l}\text { Blastocyst } \\
\text { stage }\end{array}$ & $\begin{array}{l}\text { Cleavage } \\
\text { stage }\end{array}$ & & & & \\
\hline $\begin{array}{l}\mathrm{Li} \\
\quad(2017)^{32}\end{array}$ & 2014-2015 & China & 371 & 281 & Retro & Fresh & $\begin{array}{l}\text { Data extracted from } \\
\text { database of ART Center } \\
\text { in Northwest Women's } \\
\text { and Children's Hospital }\end{array}$ & PTB, LBW \\
\hline $\begin{array}{l}\text { Pereira } \\
\qquad(2016)^{34}\end{array}$ & 2004-2013 & USA & 70 & 709 & Retro & Fresh & $\begin{array}{l}\text { Data extracted from } \\
\text { Ronald O. Perelman } \\
\text { and Claudia Cohen } \\
\text { Center for Reproductive } \\
\text { Medicine }\end{array}$ & $\begin{array}{l}\text { PTB, LBW, } \\
\text { VLBW }\end{array}$ \\
\hline $\begin{array}{l}\text { Ginström } \\
\text { Ernstad } \\
(2016)^{10}\end{array}$ & $2002-2013$ & Sweden & 4819 & 25747 & Retro & $\begin{array}{l}\text { Fresh }+ \\
\quad \text { frozen* }\end{array}$ & $\begin{array}{l}\text { In-vitro fertilization } \\
\text { register cross-linked } \\
\text { with Swedish Medical } \\
\text { Birth Register, Register } \\
\text { of Birth Defects and } \\
\text { National Patient } \\
\text { Register }\end{array}$ & $\begin{array}{l}\text { CA, LBW, } \\
\text { LGA, PM, } \\
\text { PTB, SGA } \\
\text { VPTB, VLBW }\end{array}$ \\
\hline $\begin{array}{l}\text { Chambers } \\
(2015)^{9}\end{array}$ & 2009-2012 & $\begin{array}{l}\text { Australia } \\
\text { and } \\
\text { New } \\
\text { Zealand }\end{array}$ & 28615 & 15337 & Retro & $\begin{array}{l}\text { Fresh }+ \\
\quad \text { frozen } \dagger\end{array}$ & $\begin{array}{l}\text { Data extracted from } \\
\text { ANZARD, a } \\
\text { population-based } \\
\text { registry of all ART } \\
\text { cycles undertaken in } \\
\text { Australia and New } \\
\text { Zealand }\end{array}$ & LBW, PTB, SGA \\
\hline $\begin{array}{l}\text { De Vos } \\
\qquad(2015)^{29}\end{array}$ & 2004-2009 & Belgium & 864 & 1234 & Retro & Fresh & $\begin{array}{l}\text { Centre for Reproductive } \\
\text { Medicine, Universitair } \\
\text { Ziekenhuis Brussel }\end{array}$ & $\begin{array}{l}\text { LBW, PTB, } \\
\text { VPTB, VLBW }\end{array}$ \\
\hline $\begin{array}{l}\text { Maxwell } \\
\qquad(2015)^{2}\end{array}$ & 2003-2012 & USA & 1484 & 377 & Retro & Fresh & $\begin{array}{l}\text { New York University } \\
\text { Fertility Center data }\end{array}$ & РTB, VPTB \\
\hline $\begin{array}{l}\text { Ishihara } \\
\qquad(2014)^{12}\end{array}$ & $2008-2010$ & Japan & 33389 & 14769 & Retro & $\begin{array}{l}\text { Fresh }+ \\
\quad \text { frozen* }\end{array}$ & $\begin{array}{l}\text { Japanese nationwide } \\
\text { registry of ART }\end{array}$ & $\begin{array}{l}\text { LBW, LGA, } \\
\text { PTB, SGA, } \\
\text { VPTB, VLBW }\end{array}$ \\
\hline $\begin{array}{l}\text { Oron } \\
\qquad(2014)^{24}\end{array}$ & $2008-2012$ & Canada & 94 & 279 & Retro & Fresh & $\begin{array}{l}\text { Computerized database of } \\
\text { McGill University } \\
\text { Health Center; detailed } \\
\text { telephone survey } \\
\text { conducted by trained } \\
\text { personnel }\end{array}$ & $\begin{array}{l}\text { CA, LBW, LGA, } \\
\text { PTB, SGA, } \\
\text { VLBW, VPTB }\end{array}$ \\
\hline $\begin{array}{l}\text { Zhu } \\
\qquad(2014)^{36}\end{array}$ & 2009-2012 & China & 96 & 2883 & Retro & Fresh & $\begin{array}{l}\text { Reproductive Medical } \\
\text { Center of Peking } \\
\text { University Third } \\
\text { Hospital data }\end{array}$ & LGA, SGA \\
\hline $\begin{array}{l}\text { Dar } \\
\qquad(2013)^{28}\end{array}$ & 2001-2009 & Canada & 3206 & 9506 & Retro & Fresh & $\begin{array}{l}\text { Data from large Canadian } \\
\text { cohort, Canadian ART } \\
\text { Register (CARTR) }\end{array}$ & $\begin{array}{l}\text { CA, LBW, PM, } \\
\text { PTB, VPTB, } \\
\text { VLBW }\end{array}$ \\
\hline $\begin{array}{l}\text { Fernando } \\
\qquad(2012)^{30}\end{array}$ & 2004-2009 & Australia & 1716 & 2486 & Retro & $\begin{array}{l}\text { Fresh }+ \\
\quad \text { frozen } †\end{array}$ & Monash IVF database & $\begin{array}{l}\text { LBW, LGA, } \\
\text { PTB, SGA, } \\
\text { VLBW, VPTB }\end{array}$ \\
\hline $\begin{array}{l}\text { Kalra } \\
\qquad(2012)^{31}\end{array}$ & $2004-2006$ & USA & 14746 & 32377 & Retro & Fresh & $\begin{array}{l}\text { Society of Assisted } \\
\text { Reproductive } \\
\text { Technologies database }\end{array}$ & $\begin{array}{l}\text { LBW, PTB, } \\
\text { SGA, VPTB }\end{array}$ \\
\hline $\begin{array}{l}\text { Martin } \\
\qquad(2012)^{33}\end{array}$ & $2002-2009$ & France & 588 & 959 & Retro & Fresh & $\begin{array}{l}\text { FIVNAT forms, filled in } \\
\text { voluntarily by couples }\end{array}$ & CA, PTB, PM \\
\hline $\begin{array}{l}\text { Schwarzler } \\
(2004)^{35}\end{array}$ & 1999-2001 & Austria & 173 & 100 & Retro & Fresh & $\begin{array}{l}\text { University of Innsbruck } \\
\text { data }\end{array}$ & LBW, PTB \\
\hline
\end{tabular}

Only first author of each study is given. "Data from fresh cycles and from frozen cycles analyzed separately. $\nmid$ Data from fresh and frozen cycles combined (mixed). ART, assisted reproductive technology; CA, congenital anomalies; FIVNAT, Fécondation In Vitro National; IVF, in-vitro fertilization; LBW, low birth weight; LGA, large-for-gestational age; PM, perinatal mortality; PTB, preterm birth; Retro, retrospective; SGA, small-for-gestational age; VLBW, very low birth weight; VPTB, very preterm birth. 
Table 2 Quality assessment of included studies according to Newcastle-Ottawa Scale

\begin{tabular}{|c|c|c|c|c|}
\hline Study & Selection & Comparability & Outcome & Total score \\
\hline $\operatorname{Li}(2017)^{32}$ & $* * *$ & $*$ & $* * *$ & 7 \\
\hline Pereira $(2016)^{34}$ & $* *$ & $*$ & $* * *$ & 6 \\
\hline Ginström Ernstad $(2016)^{10}$ & $* * * *$ & $* *$ & $* * *$ & 9 \\
\hline Chambers $(2015)^{9}$ & $* * * *$ & $* *$ & $* * *$ & 9 \\
\hline De Vos $(2015)^{29}$ & $* * *$ & $*$ & $* * *$ & 7 \\
\hline Maxwell $(2015)^{2}$ & $* * *$ & $*$ & $* * *$ & 7 \\
\hline Ishihara $(2014)^{12}$ & $* * * *$ & $* *$ & $* * *$ & 9 \\
\hline Oron $(2014)^{24}$ & $* * *$ & $*$ & $* * *$ & 7 \\
\hline Zhu $(2014)^{36}$ & $* * *$ & $*$ & $* * *$ & 7 \\
\hline $\operatorname{Dar}(2013)^{28}$ & $* * * *$ & $* *$ & $* * *$ & 9 \\
\hline Fernando $(2012)^{30}$ & $* *$ & $* *$ & $* * *$ & 7 \\
\hline Kalra $(2012)^{31}$ & $* * * *$ & $* *$ & $* * *$ & 9 \\
\hline $\operatorname{Martin}(2012)^{33}$ & $* * * *$ & $*$ & $* *$ & 7 \\
\hline Schwarzler $(2004)^{35}$ & $* *$ & $*$ & $* * *$ & 6 \\
\hline
\end{tabular}

Only first author of each study is given.

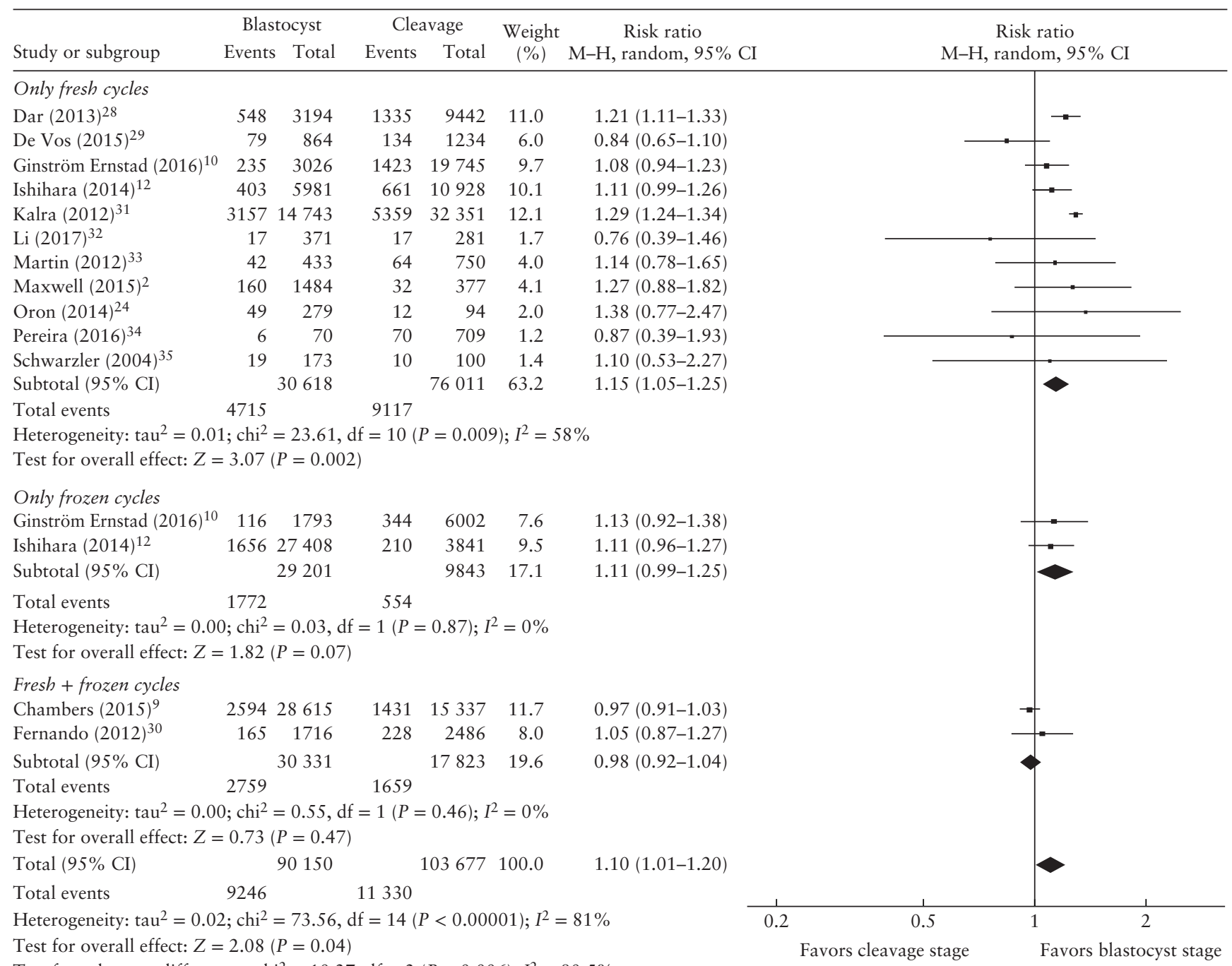

Figure 2 Forest plot of risk ratio for preterm birth before 37 weeks in singleton pregnancy resulting from blastocyst- $v s$ cleavage-stage embryo transfer. In addition to overall summary, subgroup analyses of fresh, frozen and fresh plus frozen cycles are presented. Only first author of each study is given. M-H, Mantel-Haenszel. 


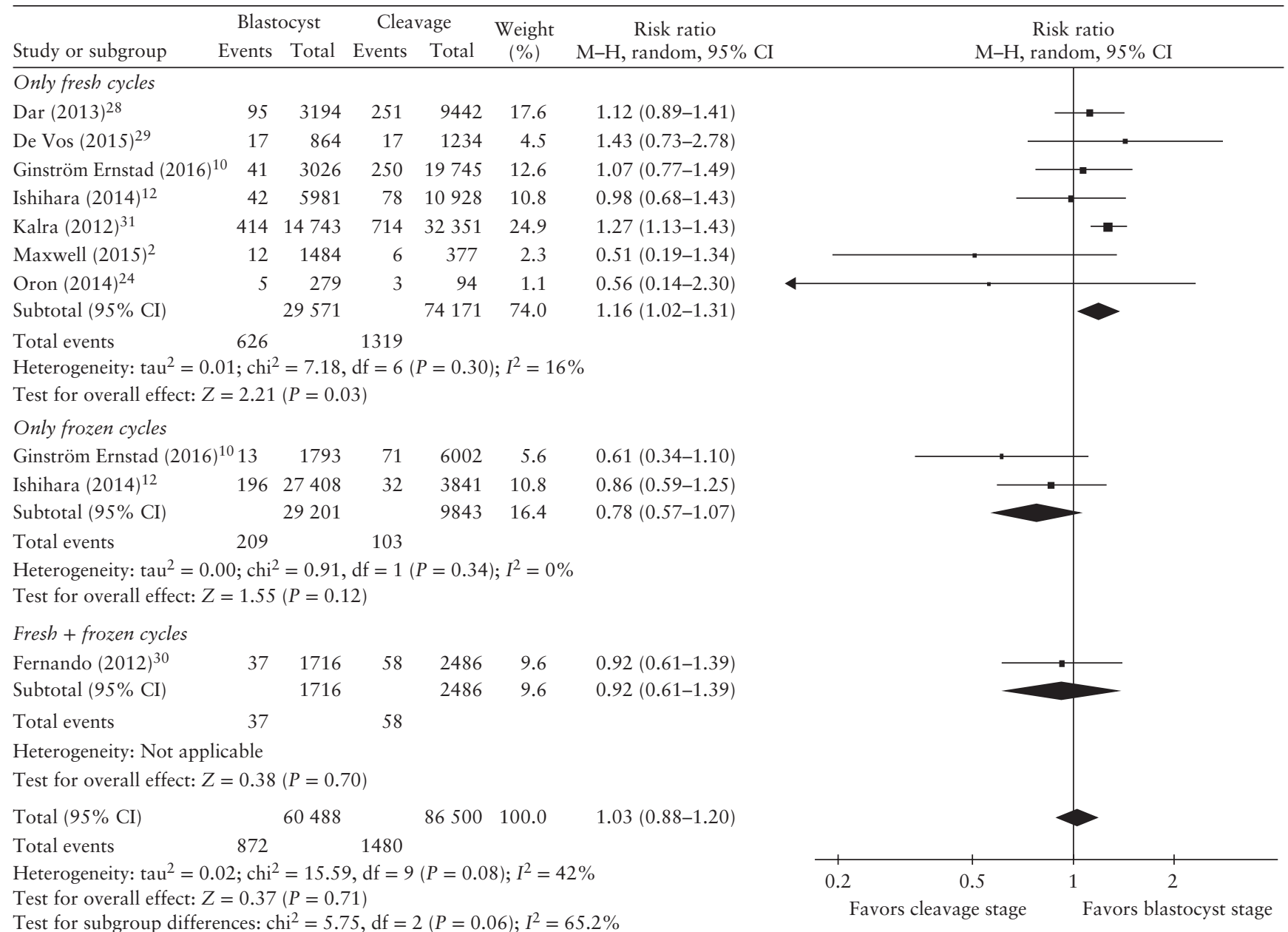

Figure 3 Forest plot of risk ratio for very preterm birth before 32 weeks in singleton pregnancy resulting from blastocyst- $v s$ cleavage-stage embryo transfer. In addition to overall summary, subgroup analyses of fresh, frozen and fresh plus frozen cycles are presented. Only first author of each study is given. M-H, Mantel-Haenszel.

cleavage stage, $n=45929)$. A higher risk of LGA birth in blastocyst- than in cleavage-stage transfers was revealed by the overall effect size (RR, $1.12(95 \%$ CI, $1.03-1.21) ; P=0.005, I^{2}=40 \%$ ) and by subgroup analysis of frozen cycles (RR, 1.18 (95\% CI, 1.09-1.27); $P<0.0001, I^{2}=0 \%$ ) (Figure 5). Subgroup analysis of fresh cycles and fresh plus frozen cycles data did not show significant differences between blastocyst- and cleavage-stage transfers with respect to LGA.

The incidence of perinatal mortality was assessed in three studies ${ }^{10,28,33}$ (blastocyst stage, $n=8458$; cleavage stage, $n=36003)$. A significantly higher risk of perinatal mortality in blastocyst- than in cleavage-stage pregnancies was revealed by the overall effect size (RR, 1.48 (95\% CI, 1.11-1.97); $P=0.008, I^{2}=0 \%$ ) and subgroup analysis of the only study that reported data from frozen cycles alone $^{10}$ (RR, 1.80 (95\% CI, 1.07-3.01); $P=0.03$ ) (Figure 6). Subgroup analysis of fresh cycles showed a similar risk of perinatal mortality after both blastocystand cleavage-stage embryo transfer.

Very low birth weight $(<1500 \mathrm{~g})$ was evaluated in seven studies $^{10,12,24,28-30,34}$ (blastocyst stage, $n=44122$; cleavage stage, $n=54148$ ) and the incidence of congenital anomalies in four studies ${ }^{10,24,28,33}$ (blastocyst stage, $n=8737$; cleavage stage, $n=36097)$. There was no significance difference in the rate of very low birth weight or congenital anomalies between blastocyst-stage and cleavage-stage transfers, irrespective of the cryopreservation procedure used (Figures S2 and S3).

\section{Risk of bias and quality of evidence}

The risk of bias across studies was minimized by a comprehensive search for eligible studies and exclusion of duplicate data. Visual inspection of funnel plots showed no publication bias in terms of primary outcomes (preterm birth Egger's test, $P=0.62$; low birth weight Egger's test, $P=0.93$ ) (Figure S4).

Sensitivity analysis, excluding studies with a high risk of bias, revealed that the pooled effect sizes were not affected (Table S1). The quality of evidence using the GRADE scoring system is reported in Table S2.

\section{DISCUSSION}

\section{Summary of evidence}

Recent systematic reviews comparing perinatal and neonatal outcomes in singleton pregnancies from 


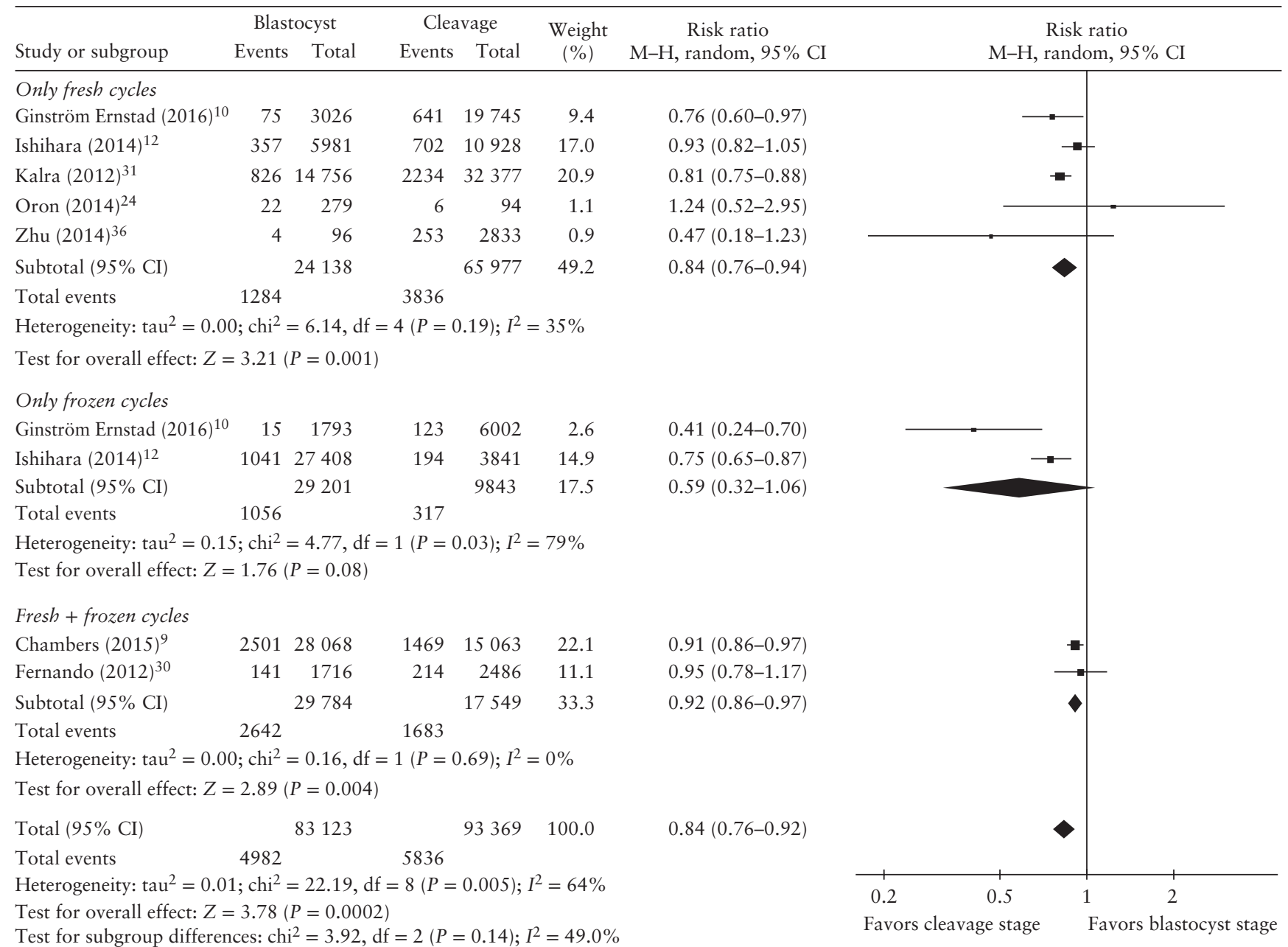

Figure 4 Forest plot of risk ratio for small-for-gestational-age neonates in singleton pregnancy resulting from blastocyst- $v s$ cleavage-stage embryo transfer. In addition to overall summary, subgroup analyses of fresh, frozen and fresh plus frozen cycles are presented. Only first author of each study is given. M-H, Mantel-Haenszel.

cleavage-stage and from blastocyst-stage embryos have raised concerns about the safety of extended embryo culture in $\mathrm{IVF}^{8,11,14}$. According to these studies, pregnancies following blastocyst-stage embryo transfer are associated with an increased risk of preterm birth, very preterm birth and LGA, and a reduced risk of SGA. However, the differences observed in our study with respect to perinatal outcomes between blastocystand cleavage-stage embryo transfer in fresh and frozen cycles suggest that cryopreservation of the embryos and extended embryo culture could have affected previous results.

Our results regarding fresh cycles are consistent with those reported by Martins et al. ${ }^{14}$ in terms of preterm birth, very preterm birth and SGA. In fact, we detected a higher incidence of preterm birth but a lower number of SGA births in blastocyst- than in cleavage-stage pregnancies. However, when considering fresh plus frozen cycles and frozen only cycles, there was no difference in the rate of very preterm birth between blastocyst- and cleavage-stage pregnancies (Figure 3). The rates of low birth weight, very low birth weight and congenital anomalies were similar after blastocyst-stage and cleavage-stage embryo transfer, irrespective of the cryopreservation procedure.

A trend towards a higher risk of preterm birth in fresh $v$ s frozen cycles was confirmed by Ginström Ernstad et al..$^{10}$. In fact, for both fresh blastocyst- and cleavage-stage cycles, there was a trend towards a higher risk of preterm birth than for frozen blastocyst- and cleavage-stage cycles $(7.8 \%$ with fresh blastocysts vs $6.5 \%$ with frozen blastocysts, $P=0.09 ; 7.2 \%$ with fresh cleavage stage vs $5.2 \%$ with frozen cleavage stage, $P<0.05)$. Conversely, the risk of LGA differed significantly between blastocyst-stage and cleavage-stage embryos in frozen but not in fresh cycles. This observation is supported by previous studies that demonstrated a higher incidence of LGA in frozen cycles than in fresh cycles ${ }^{37}$. The different risks of preterm birth in frozen $v$ s fresh cycles might be explained by at least two factors. Firstly, synchronization between the endometrium and embryos at the time of transfer might be more accurate in frozen cycles ${ }^{38}$. Secondly, the supraphysiological hormonal conditions produced by ovarian stimulation in fresh cycles may affect many aspects, namely early conception, peri-implantation function and development, all of which in turn might 


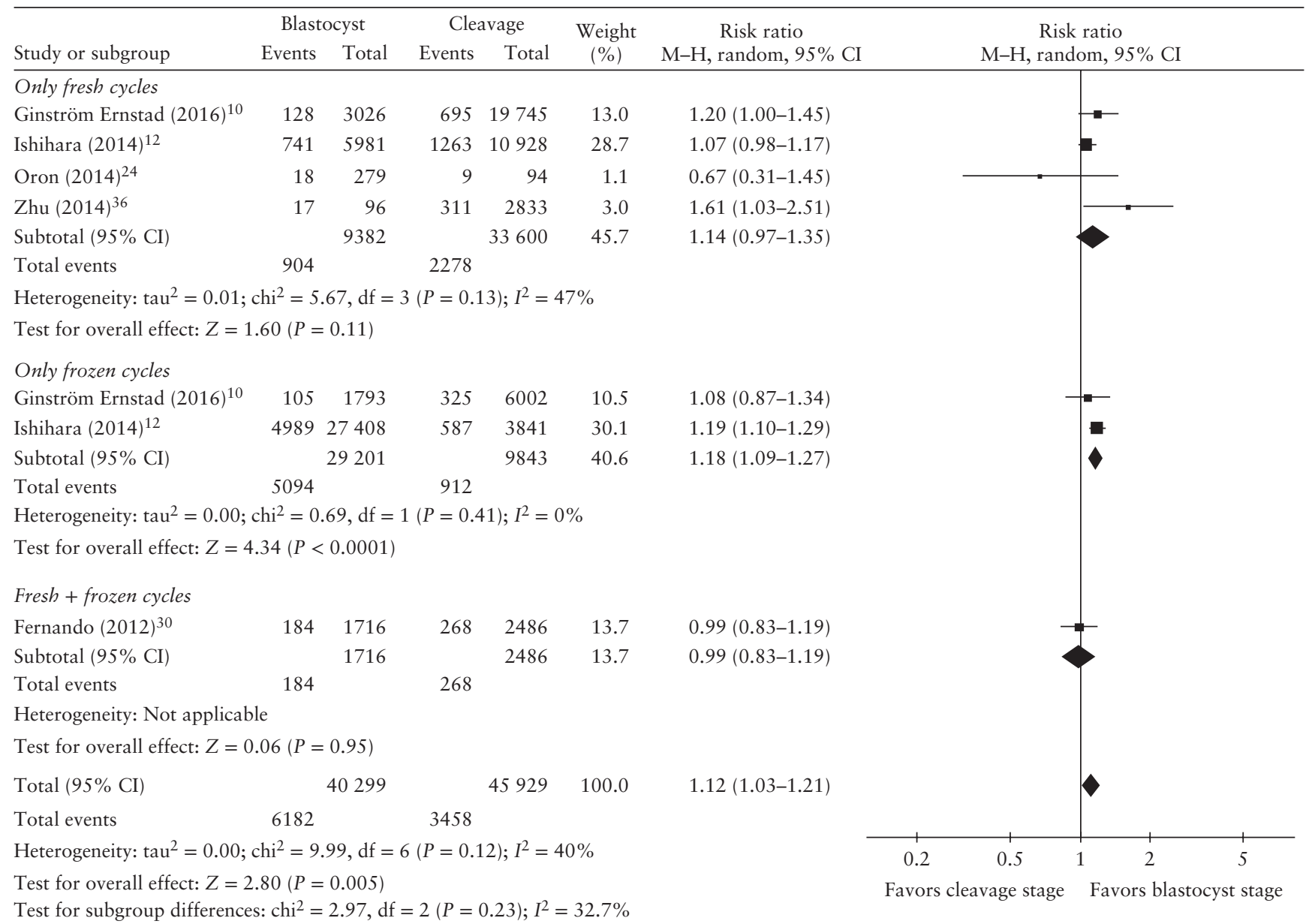

Figure 5 Forest plot of risk ratio for large-for-gestational-age neonates in singleton pregnancy resulting from blastocyst- $v s$ cleavage-stage embryo transfer. In addition to overall summary, subgroup analyses of fresh, frozen and fresh plus frozen cycles are presented. Only first author of each study is given. M-H, Mantel-Haenszel.

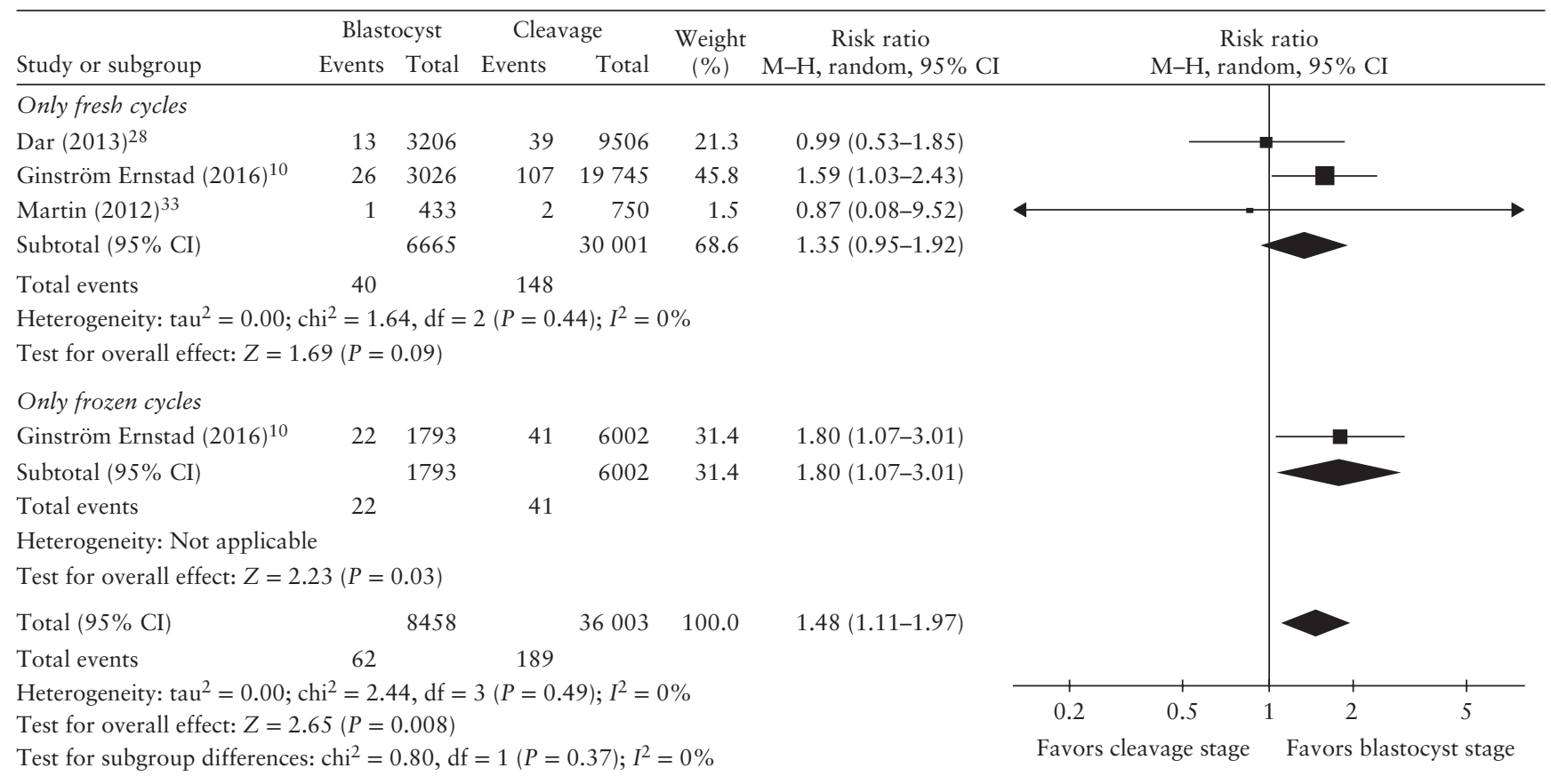

Figure 6 Forest plot of risk ratio for perinatal mortality in singleton pregnancy resulting from blastocyst- $v s$ cleavage-stage embryo transfer. In addition to overall summary, subgroup analyses of fresh only and frozen only cycles are presented. Only first author of each study is given. M-H, Mantel-Haenszel. 
influence the time of delivery ${ }^{39,40}$. Furthermore, the higher intra-abdominal volume and inflammatory conditions secondary to continuous ovarian stimulation might explain the higher rates of preterm and very preterm delivery after fresh cycle embryo transfer.

The risk of SGA birth was significantly lower after transfer of blastocysts than after transfer of cleavage-stage embryos in fresh cycles and when fresh and frozen cycles were considered collectively. In frozen cycles, there was a near significant reduction $(P=0.08)$ of SGA births in blastocyst- $v$ s cleavage-stage pregnancies. Data regarding the effect of frozen cycles on SGA are contradictory; some authors reported a reduced incidence of SGA after frozen embryo transfer than after fresh embryo transfer ${ }^{41}$, whereas others did not ${ }^{42}$.

We did not observe any significant difference in the risk of perinatal mortality between blastocyst- and cleavage-stage pregnancies in fresh cycles; however, the only study that reported data from frozen cycles reported an increased risk after blastocyst transfer ${ }^{10}$. In contrast to the findings of Dar et al. ${ }^{8}$ and Kallen et al. ${ }^{21}$, we did not observe a higher risk of congenital anomalies after blastocyst-stage embryo transfer. This discrepancy may reflect the improvements made in IVF techniques and embryo culture media ${ }^{10}$. Indeed, data from the same register over time showed that the higher risk of congenital malformations after blastocyst transfer reported by Kallen et al. ${ }^{21}$ was not observed in a subsequent study conducted by Ginström Ernstad et al. ${ }^{10}$.

Although the risk of preterm birth and very preterm birth associated with the transfer of blastocyst-stage embryos could cause concern, this procedure has advantages, namely a higher implantation rate, the possibility of selecting the most viable embryos, thereby minimizing the risk of transfer failure, and better synchronization between the endometrium and embryos at the time of transfer ${ }^{43}$. Furthermore, extending embryo culture could favor a single embryo transfer policy, which is recommended to ensure live births for infertile couples and to reduce the risk of multiple pregnancies ${ }^{44}$. However, these advantages were recently questioned by Martins et $a l^{7}{ }^{7}$, and we concur with them that more well designed randomized trials are needed before a definitive conclusion can be drawn regarding this issue.

Finally, the fact that frozen cycles could somehow mitigate the adverse effect of extended embryo culture on perinatal outcomes could encourage the practice of cryopreservation in the clinical setting. This strategy has the advantage of reducing ovarian hyperstimulation syndrome and of limiting the number of controlled ovarian stimulations required to achieve pregnancy 45 .

\section{Limitations of the study}

The main limitation of this study is the low quality of data available. In fact, only retrospective studies were available. Furthermore, although cryopreservation appeared to influence perinatal outcomes, further studies evaluating frozen cycles are necessary to verify whether this is indeed the case. Although we recognize that this strategy could increase Type-I and -II errors, data from frozen cycles were collected from the largest and better quality studies with more than 30000 observations in terms of preterm birth, very preterm birth, SGA, LGA, low birth weight and very low birth weight ${ }^{10,12}$. Regarding the other outcomes, namely perinatal mortality and congenital anomalies, our findings are limited because our analysis was restricted to only one study that reported data from only frozen cycles ${ }^{10}$. Lastly, the definitions of SGA and LGA are not consistent throughout the studies included in this meta-analysis, and depend largely on the type of growth chart employed ${ }^{9,10,12}$, ethnic and racial characteristics and on the cut-off values adopted. Finally, we were unable to determine whether the procedure used for cryopreservation affected our results.

\section{Conclusions}

In fresh cycles, the risk of preterm and very preterm birth was significantly higher after blastocyst-stage than after cleavage-stage embryo transfer. However, this risk was comparable when frozen cycles were included in the analysis. Conversely, while no differences were observed in fresh cycles, LGA births were more frequent in blastocyst- $v s$ cleavage-stage embryo transfer with frozen cycles. SGA deliveries were significantly fewer after blastocyst- than after cleavage-stage transfer in fresh cycles and a similar trend, albeit not statistically significant, was observed in frozen cycles. No differences were observed with respect to low birth weight, very low birth weight and congenital anomalies, irrespective of cryopreservation procedure. Only one study reported a higher risk of perinatal mortality in blastocyst-stage $v s$ cleavage-stage pregnancies in frozen cycles. Caution should be exercised in interpreting the findings reported here, given the low level of evidence available and the wide heterogeneity of studies included in the analysis.

\section{ACKNOWLEDGMENT}

We thank Jean Gilder (Scientific Communication srl., Naples, Italy) for revising and editing the manuscript.

\section{REFERENCES}

1. Hardarson T, Van Landuyt L, Jones G. The blastocyst. Hum Reprod 2012; 27 (Suppl 1): i72-i91.

2. Maxwell SM, Melzer-Ross K, McCulloh DH, Grifo JA. A comparison of pregnancy outcomes between day 3 and day $5 / 6$ embryo transfers: does day of embryo transfer really make a difference? J Assist Reprod Genetics 2015; 32: 249-254.

3. Capalbo A, Ubaldi FM, Cimadomo D, Maggiulli R, Patassini C, Dusi L, Sanges F, Buffo L, Venturella R, Rienzi L. Consistent and reproducible outcomes of blastocyst biopsy and aneuploidy screening across different biopsy practitioners: a multicentre study involving 2586 embryo biopsies. Hum Reprod 2016; 31: 199-208.

4. Ubaldi FM, Capalbo A, Vaiarelli A, Cimadomo D, Colamaria S, Alviggi C, Trabucco E, Venturella R, Vajta G, Rienzi L. Follicular versus luteal phase ovarian stimulation during the same menstrual cycle (DuoStim) in a reduced ovarian reserve population results in a similar euploid blastocyst formation rate: new insight in ovarian reserve exploitation. Fertil Steril 2016; 105: 1488-1495.e1.

5. Munne S, Sandalinas M, Escudero T, Marquez C, Cohen J. Chromosome mosaicism in cleavage-stage human embryos: evidence of a maternal age effect. Reprod Biomed Online 2002; 4: 223-232.

6. Glujovsky D, Farquhar C, Quinteiro Retamar AM, Alvarez Sedo CR, Blake D Cleavage stage versus blastocyst stage embryo transfer in assisted reproductive technology. Cochrane Database Syst Rev 2016; 6: CD002118. 
7. Martins WP, Nastri CO, Rienzi L, van der Poel SZ, Gracia C, Racowsky C. Blastocyst vs cleavage-stage embryo transfer: systematic review and meta-analysis of reproductive outcomes. Ultrasound Obstet Gynecol 2017; 49: 583-591.

8. Dar S, Lazer T, Shah PS, Librach CL. Neonatal outcomes among singleton births after blastocyst versus cleavage stage embryo transfer: a systematic review and meta-analysis. Hum Reprod Update 2014; 20: 439-448.

9. Chambers GM, Chughtai AA, Farquhar CM, Wang YA. Risk of preterm birth after blastocyst embryo transfer: a large population study using contemporary registry data from Australia and New Zealand. Fertil Steril 2015; 104: 997-1003.

10. Ginström Ernstad E, Bergh C, Khatibi A, Källén KB, Westlander G, Nilsson S, Wennerholm UB. Neonatal and maternal outcome after blastocyst transfer: population-based registry study. Am J Obstet Gynecol 2016; 214: 378.e1-378.e10.

11. Maheshwari A, Kalampokas T, Davidson J, Bhattacharya S. Obstetric and perinatal outcomes in singleton pregnancies resulting from the transfer of blastocyst-stage versus cleavage-stage embryos generated through in vitro fertilization treatment: a systematic review and meta-analysis. Fertil Steril 2013; 100: 1615-1621.e1-10.

12. Ishihara O, Araki R, Kuwahara A, Itakura A, Saito H, Adamson GD. Impact of frozen-thawed single-blastocyst transfer on maternal and neonatal outcome: an analysis of 277,042 single-embryo transfer cycles from 2008 to 2010 in Japan. Fertil Steril 2014; 101: 128-133.

13. Oron G, Nayot D, Son WY, Holzer H, Buckett W, Tulandi T. Obstetric and perinatal outcome from single cleavage transfer and single blastocyst transfer: a matched case-control study. Gynecol Endocrinol 2015; 31: 469-472.

14. Martins WP, Nastri CO, Rienzi L, van der Poel SZ, Gracia CR, Racowsky C. Obstetrical and perinatal outcomes following blastocyst transfer compared to cleavage transfer: a systematic review and meta-analysis. Hum Reprod 2016; 31: 2561-2569.

15. Wells G, Shea B, O'connell D, Peterson J, Welch V, Losos M, Tugwell P. Quality Assessment Scales for Observational Studies. Ottawa Health Research Institute 2004.

16. Duval S. The Trim and Fill Method. In Publication Bias in Meta-Analysis: Prevention, Assessment and Adjustments. Rothstein HR, Sutton AJ, Borenstein M (eds). J. Wiley \& Sons, Ltd: Chichester, UK, 2006; 127-144.

17. Egger M, Davey Smith G, Schneider M, Minder C. Bias in meta-analysis detected by a simple, graphical test. BMJ 1997; 315: 629-634.

18. Guyatt G, Oxman AD, Akl EA, Kunz R, Vist G, Brozek J, Norris S, Falck-Ytter Y, Glasziou P, DeBeer H, Jaeschke R, Rind D, Meerpohl J, Dahm P, Schünemann HJ. GRADE guidelines: 1. Introduction - GRADE evidence profiles and summary of findings tables. J Clin Epidemiol 2011; 64: 383-394.

19. Wang YA, Costello M, Chapman M, Black D, Sullivan EA. Transfers of fresh blastocysts and blastocysts cultured from thawed cleavage embryos are associated with fewer miscarriages. Reprod Biomed Online 2011; 23: 777-788.

20. Sotiroska V, Petanovski Z, Dimitrov G, Hadji-Lega M, Shushleski D, Saltirovski S, Matevski V, Shenbakar S, Panov S, Johansson L. The day of embryo transfer affects delivery rate, birth weights, female-to-male ratio, and monozygotic twin rate. Taiwan J Obstet Gynecol 2015; 54: 716-721.

21. Kallen B, Finnstrom O, Lindam A, Nilsson E, Nygren KG, Olausson PO. Blastocyst versus cleavage stage transfer in in vitro fertilization: differences in neonatal outcome? Fertil Steril 2010; 94: 1680-1683.

22. Nakashima A, Araki R, Tani H, Ishihara O, Kuwahara A, Irahara M, Yoshimura Y, Kuramoto T, Saito H, Nakaza A, Sakumoto T. Implications of assisted reproductive technologies on term singleton birth weight: an analysis of 25,777 children in the national assisted reproduction registry of Japan. Fertil Steril 2013; 99: 450-455.

23. Sazonova A, Kallen K, Thurin-Kjellberg A, Wennerholm UB, Bergh C. Factors affecting obstetric outcome of singletons born after IVF. Hum Reprod 2011; 26: 2878-2886.

24. Oron G, Sokal-Arnon T, Son WY, Demirtas E, Buckett W, Zeadna A, Holzer H, Tulandi T. Extended embryo culture is not associated with increased adverse obstetric or perinatal outcome. Am J Obstet Gynecol 2014; 211: 165.e1-7.

25. Mäkinen S, Söderström-Anttila V, Vainio J, Suikkari AM, Tuuri T. Does long in vitro culture promote large for gestational age babies? Hum Reprod 2013; 28: 828-834.
26. Wikland M, Hardarson T, Hillensjo T, Westin C, Westlander G, Wood M, Wennerholm UB Obstetric outcomes after transfer of vitrified blastocysts. Hum Reprod 2010; 25: 1699-1707.

27. Kaartinen N, Das P, Kananen K, Huhtala H, Tinkanen H. Can repeated IVF-ICSI-cycles be avoided by using blastocysts developing from poor-quality cleavage stage embryos? Reprod Biomed Online 2015; 30: 241-247.

28. Dar S, Librach CL, Gunby J, Bissonnette F, Cowan L. Increased risk of preterm birth in singleton pregnancies after blastocyst versus Day 3 embryo transfer: Canadian ART Register (CARTR) analysis. Hum Reprod 2013; 28: 924-928.

29. De Vos A, Janssens R, Van de Velde H, Haentjens P, Bonduelle M, Tournaye H, Verheyen $\mathrm{G}$. The type of culture medium and the duration of in vitro culture do not influence birthweight of ART singletons. Hum Reprod 2015; 30: 20-27.

30. Fernando D, Halliday JL, Breheny S, Healy DL. Outcomes of singleton births after blastocyst versus nonblastocyst transfer in assisted reproductive technology. Fertil Steril 2012; 97: 579-584.

31. Kalra SK, Ratcliffe SJ, Barnhart KT, Coutifaris C. Extended embryo culture and an increased risk of preterm delivery. Obstet Gynecol 2012; 120: 69-75.

32. Li W, Xue X, Zhao W, Ren A, Zhuo W, Shi J. Blastocyst transfer is not associated with increased unfavorable obstetric and perinatal outcomes compared with cleavage-stage embryo transfer. Gynecol Endocrinol 2017: 33: 857-860.

33. Martin L, Frapsauce C, Royère D, Guérif F. [Single pregnancy outcome afte blastocyst transfer: comparison with cleavage stage embryo transfers]. Gynecol Obstet Fertil 2012; 40: 291-295.

34. Pereira N, Pryor KP, Petrini AC, Lekovich JP, Stahl J, Elias RT, Spandorfer SD. Perinatal Risks Associated with Early Vanishing Twin Syndrome following Transfer of Cleavage- or Blastocyst-Stage Embryos. I Pregnancy 2016; 2016 1245210.

35. Schwarzler P, Zech H, Auer M, Pfau K, Gobel G, Vanderzwalmen P, Zech N Pregnancy outcome after blastocyst transfer as compared to early cleavage stage embryo transfer. Hum Reprod 2004; 19: 2097-2102.

36. Zhu JL, Lin SL, Li M, Chen LX, Lian Y, Liu P, Qiao J. Effect of in vitro culture period on birthweight of singleton newborns. Hum Reprod 2014; 29: 448-454.

37. Pinborg A, Henningsen AA, Loft A, Malchau SS, Forman J, Andersen AN. Large baby syndrome in singletons born after frozen embryo transfer (FET): is it due to maternal factors or the cryotechnique? Hum Reprod 2014; 29: 618-627.

38. Ming L, Liu P, Qiao J, Lian Y, Zheng X, Ren X, Huang J, Wu Y. Synchronization between embryo development and endometrium is a contributing factor for rescue ICSI outcome. Reprod Biomed Online 2012; 24: 527-531.

39. Farhi J, Ben-Haroush A, Andrawus N, Pinkas H, Sapir O, Fisch B, Ashkenazi J. High serum oestradiol concentrations in IVF cycles increase the risk of pregnancy complications related to abnormal placentation. Reprod Biomed Online 2010; 21: $331-337$.

40. Ozgur K, Berkkanoglu M, Bulut H, Humaidan P, Coetzee K. Perinatal outcomes after fresh versus vitrified-warmed blastocyst transfer: retrospective analysis. Fertil Steril 2015; 104: 899-907.e3.

41. Kato O, Kawasaki N, Bodri D, Kuroda T, Kawachiya S, Kato K, Takehara Y Neonatal outcome and birth defects in 6623 singletons born following minimal ovarian stimulation and vitrified versus fresh single embryo transfer. Eur J Obstet Gynecol Reprod Biol 2012; 161: 46-50.

42. Sazonova A, Kallen K, Thurin-Kjellberg A, Wennerholm UB, Bergh C. Obstetric outcome in singletons after in vitro fertilization with cryopreserved/thawed embryos. Hum Reprod 2012; 27: 1343-1350.

43. Practice Committees of the American Society for Reproductive Medicine and the Society for Assisted Reproductive Technology. Blastocyst culture and transfer in clinical-assisted reproduction: a committee opinion. Fertil Steril 2013; 99: 667-672.

44. Practice Committee of Society for Assisted Reproductive Technology; Practice Committee of American Society for Reproductive Medicine. Elective single-embryo transfer. Fertil Steril 2012; 97: 835-842.

45. Humaidan P, Quartarolo J, Papanikolaou EG. Preventing ovarian hyperstimulation syndrome: guidance for the clinician. Fertil Steril 2010; 94: 389-400.

\section{SUPPORTING INFORMATION ON THE INTERNET}

The following supporting information may be found in the online version of this article:

Figures S1-S3 Forest plots of risk ratio for low birth weight (Figure S1), very low birth weight (Figure S2) and congenital anomalies (Figure S3) in singleton pregnancies resulting from blastocyst- $v$ s cleavage-stage embryo transfer, with subgroup analyses of fresh, frozen and fresh plus frozen cycles.

Figure S4 Funnel plots and 'trim and fill' analysis investigating publication bias in studies evaluating incidence of preterm birth (a) and low birth weight (b) in singleton pregnancies resulting from blastocyst- $v$ s cleavagestage embryo transfer.

Table S1 Sensitivity analysis excluding studies with the lowest NOS score according to cryopreservation method

Table S2 Quality of evidence according to GRADE for perinatal outcome of singleton pregnancies resulting from blastocyst- $v$ s cleavage-stage embryo transfer 